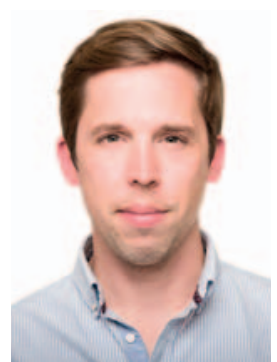

Matthias Raspe

Koordination «PneumoCampus»

\section{Weichenstellungen: Wege zur akademischen Karriere, Umgang mit der ärztlichen Schweigepflicht und ein zukunftsweisendes Urteil}

Gefühlt wurde gerade erst der Jahreswechsel gefeiert und nun ist schon der Mai angebrochen, der Frühling hat sich mittlerweile im ganzen Land ausgebreitet und wir freuen uns, Ihnen die zweite Ausgabe des «PneumoCampus» in die Hände geben zu können!

In unserem aktuellen Fall der Blickdiagnosen kommt der Patient eigentlich für eine Katarakt-Operation in die Klinik, wird dann aber bei einer bisher unbekannten Oxygenierungsstörung und Luftnot pneumologisch aufgenommen. Der Verlauf der Computertomographien des Thorax über eine Zeitspanne von insgesamt 11 Jahren zeigt das fortschreitende Bild zweier für sich allein schon schwerwiegender Erkrankungen des Lungenparenchyms. Kennen Sie die Diagnose?

Clinician Scientist-Programme werden zunehmend an Universitäten etabliert, um jungen Ärztinnen und Ärzten die Verbindung von Grundlagenforschung und klinischer Tätigkeit zu ermöglichen und den Weg einer akademischen Karriere zu erleichtern. In dieser Ausgabe stellt Prof. Nisar Malek aus Tübingen das Programm der Deutschen Gesellschaft für Innere Medizin (DGIM) e.V. vor, durch das seit 2016 erstmalig drei Stipendiatinnen und Stipendiaten über drei Jahre mit bis zu 100000 Euro unterstützt werden, um ihnen in dieser Zeit eine 50\%ige Freistellung von der Klinik zu ermöglichen.

Wissen Sie noch im Detail, ob und inwiefern Sie Patientenunterlagen bei Anfragen von Patienten selbst, von gesetzlichen Betreuern und von Ermittlungsbehörden herausgeben dürfen? In einem äußerst praxisrelevanten Beitrag erläutert Dr. sc. hum. Uwe Günther, wann Ärztinnen und Ärzte Auskunft erteilen dürfen, und wann nicht.

Als weiterer Beitrag in dieser Ausgabe gibt Caroline Siech, Verantwortliche für Presse- und Öffentlichkeitsarbeit bei der Bundesvertretung der Medizinstudierenden in Deutschland (bvmd) e.V., ein Statement zum kürzlich ergangenen Urteil des Bundesverfassungsgerichts über die Vergabe von Studienplätzen.

Ich wünsche Ihnen viel Freude beim Lesen,

Mit herzlichen Grüßen,

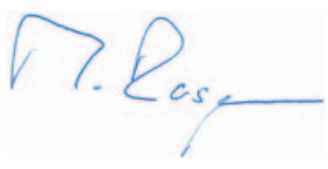

\section{KARGER}

Fax +497614520714

information@karger.com

www.karger.com (c) 2018 S. Karger GmbH, Freiburg
Dr. med. Matthias Raspe

Medizinische Klinik

Schwerpunkt Infektiologie und Pneumologie

Charité - Universitätsmedizin Berlin

Augustenburger Platz 1, 13353 Berlin, Deutschland

matthias.raspe@charite.de 


\section{Blickdiagnosen}

\section{Welche Diagnose vermuten Sie?}

Der 77-jährige Patient kam elektiv in unser Krankenhaus zur Evaluation einer Katarakt-Operation. In der Ambulanz der Ophthalmologie erlitt der Patient eine Präsynkope und wurde daraufhin in der internistischen Rettungsstelle vorgestellt, über die schließlich die Aufnahme in unsere Klinik erfolgte. Der Patient berichtet von einer langsam, vor allem über die letzten Monate zunehmenden Dyspnoe erst nur unter Belastung und zuletzt auch in Ruhe. Dazu besteht ein chronischer, meist unproduktiver Husten. Zeichen einer Infektion oder kardialen Dekompensation bestehen klinisch nicht. Der Patient ist aktiver Raucher (insgesamt ca. 40 Packungsjahre). In der Rettungsstelle fällt eine erniedrigte periphere Sauerstoffsättigung von 88\% unter Raumluft auf (Hämoglobin 10,4 g/dl). In der Lungenfunktionsdiagnostik führt eine schwere Einschränkung der
CO-Diffusionskapazität (DLCO/VA 27\% Ist/Soll). Es bestehen multiple (vor allem vaskuläre) internistische Begleiterkrankungen. Aufgrund eines Harnblasenkarzinoms wurden beim Patienten seit der Erstdiagnose 2007 wiederholt Computertomographien einschließlich des Thorax durchgeführt. Berufsanamnestisch berichtet der Patient von langjährigem Asbestkontakt während seiner früheren Tätigkeit als Maurer.

\section{Auflösung online unter: \\ www.karger.com/blickdiagnose-kkp-2-2018}

Kontaktadresse: Herrn Dr. med. Matthias Raspe, Medizinische Klinik, Schwerpunkt Infektiologie und Pneumologie, Charité - Universitätsmedizin Berlin, Augustenburger Platz 1, 13353 Berlin, Deutschland, matthias.raspe@charite.de
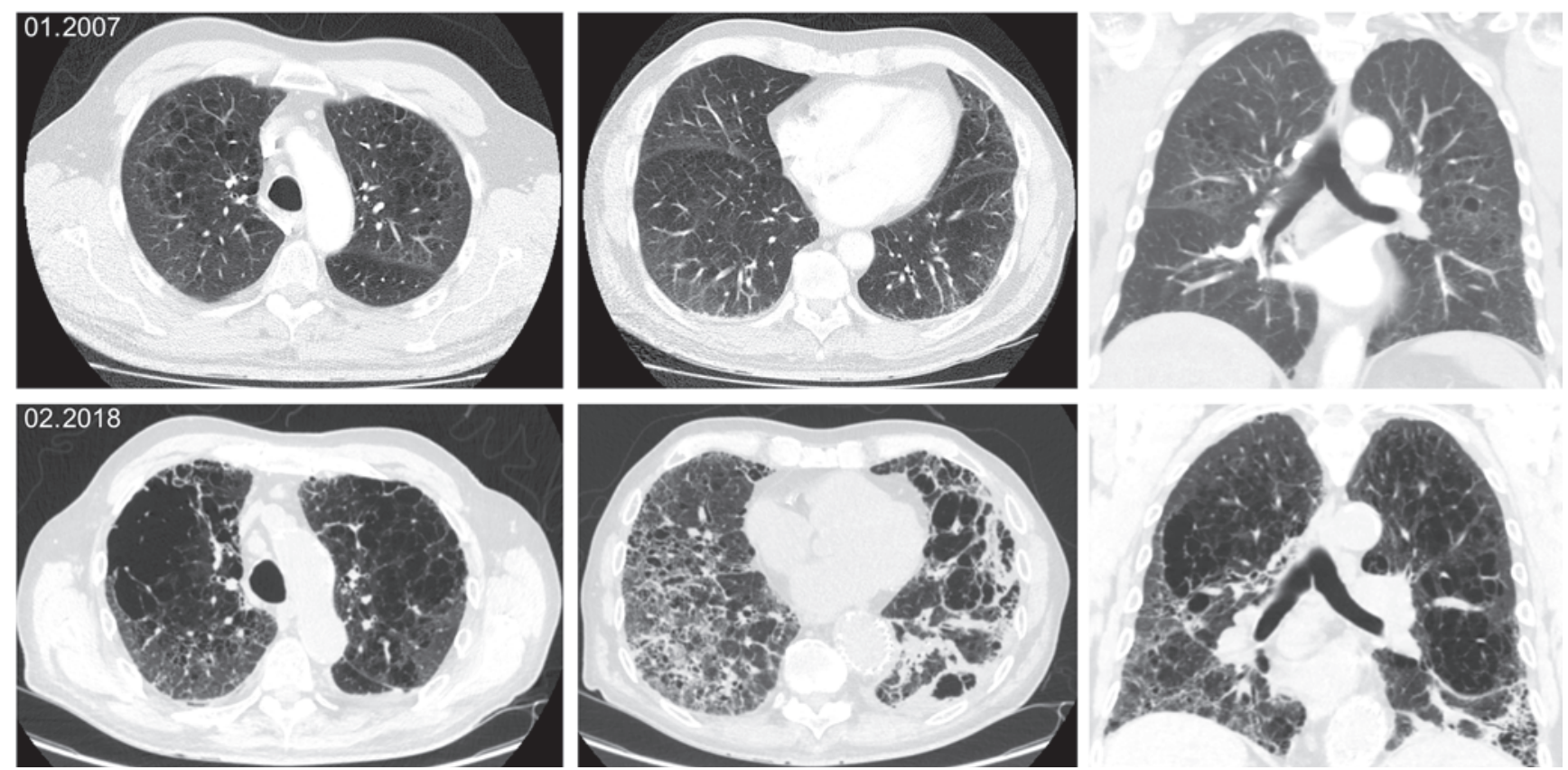

Bildbefunde mit freundlicher Genehmigung von Dr. med. C. Althoff, Leitung Interventionsradiologie Charité Campus Mitte, Institut und Klinik für Radiologie CCM und CVK, Charité - Universitätsmedizin Berlin. 
Forschung in der Weiterbildung

\section{Das Clinician Scientist-Programm der Deutschen Gesellschaft für Innere Medizin e.V. - Interview mit Prof. Dr. Nisar Malek}

Es ist wichtig, die Weiterbildungszeit nach den eigenen Vorstellungen und Präferenzen zu gestalten - denn hier werden viele Weichen gestellt. Zahlreiche Förderprogramme im In- und Ausland bieten Ärzten in Weiterbildung dafür Unterstützung. Aber welches Programm kommt für einen selbst in Frage? Um ein wenig Licht in dieses Dunkel zu bringen, gibt Prof. Dr. Nisar Malek aus Tübingen im folgenden Interview Einblicke in das Clinician Scientist-Programm der Deutschen Gesellschaft für Innere Medizin e.V.

\section{Wie ist das Clinician Scientist-Programm entstanden und welche} Intention steckt dahinter?

Das Clinician Scientist-Programm (CSP) wurde 2016 zur Förderung des wissenschaftlichen Nachwuchses in der Inneren Medizin durch die «Kommission Wissenschaft - Nachwuchsförderung» der Deutschen Gesellschaft für Innere Medizin e.V. (DGIM) aufgelegt. Intention des Förderprogramms ist die Unterstützung junger Wissenschaftler auf den verschiedenen Stufen ihres Karriereweges bis hin zur Etablierung einer eigenen Arbeitsgruppe und der erfolgreichen Habilitation. So sollen die für junge Mediziner notwendigen Freiräumen geschaffen werden, um parallel zu ihrer Aus- und Weiterbildung zum Facharzt zeitintensive, grundlagenorientierte Forschungsprojekte durchführen zu können. Die CSP-Förderung stellt je Stipendiat bis zu 100000 EUR zur Verfügung, um eine 50\%ige Freistellung für drei Jahre von den klinischen Aufgaben zu ermöglichen.

An wen richtet sich das Programm? Welche Voraussetzungen muss man mitbringen, um sich dafür zu bewerben?

Das Programm richtet sich an promovierte, wissenschaftlich interessierte Ärztinnen und Ärzte, die sich in der zweiten Hälfte ihrer Weiterbildung zum Facharzt für Innere Medizin befinden, wobei ein Forschungsprofil bereits deutlich erkennbar

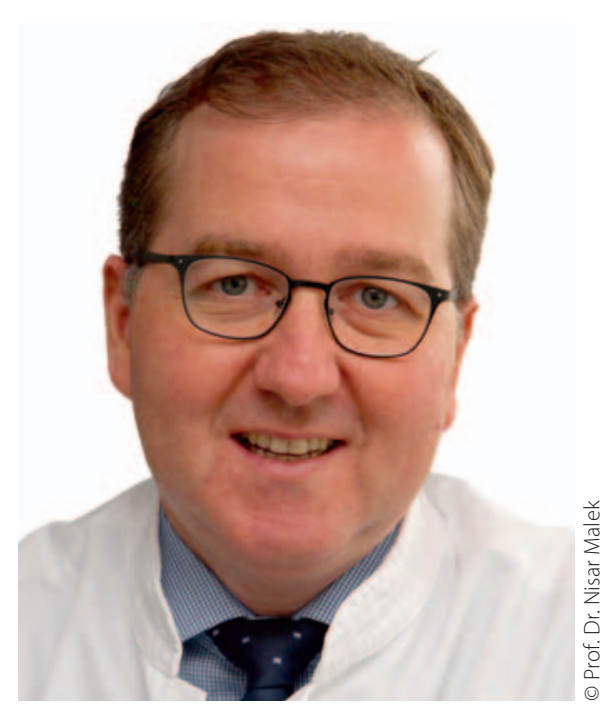

"Durch die Clinician ScientistStipendien wird die grundlagenorientierte Forschung besser in den Ausbildungsweg zum Internisten integriert.»

\section{Wie ist das Programm aufgebaut?}

Schildern Sie kurz den Ablauf.

Kernelement des CSP ist die Freistellung von klinischen Aufgaben für bis zu 50\% über maximal drei Jahre, um eine Schärfung des eigenen Forschungsprofils zu ermöglichen. In dieser Zeit kann das eigene Forschungsthema so weit etabliert werden, dass die Forschung anschließend durch Folgeanträge eigenständig weiter vorangetrieben werden kann. Das im Rahmen des CSP beantragbare Forschungsprojekt kann einen grundlagenorientierten, krankheitsorientierten oder patientenorientierten Fokus aufweisen.

Ein wesentlicher Bestandteil des Programms ist außerdem die aktive Unterstützung und Karriereförderung durch die Heimatklinik des Clinician Scientist-Stipendiaten, welche einen habilitierten Mentor mit Bezug zum Forschungsfeld zur Verfügung stellt. Dieser erklärt sich zu einer intensiven Betreuung des Clinician Scientists und regelmäßigen Mentoring-Gesprächen bereit, um sowohl die wissenschaftliche Arbeit zu begleiten als auch die ärztliche Weiterbildung zu fördern. Zum Erwerb weiterer wissenschaftlicher Kompetenzen und zur Karriereplanung wird das Programm durch ein Curriculum begleitet, dessen Kernbereich durch die Teilnahme an der DGIM-Förderakademie abgedeckt ist. Das Begleitcurriculum wird durch den Stipendiaten und dessen Mentor eigenständig erarbeitet und enthält sowohl an das eigene Forschungsfeld angepasste Maßnahmen als auch allgemeine Themengebiete, die von hoher Relevanz für die Grundlagenforschung in der Inneren Medizin sind (z.B. personalisierte Medizin, Academic Drug-Development, molekular-diagnostische Methoden, multimodale Bildgebung, Datenanalyse durch Bioinformatik und Systemmedizin etc.). Übergeordnete Fortbildungen sollen darüber hinaus Kompetenzen im Bereich Medizindidaktik und wissenschaftliches Schreiben schulen und über weitere Förderinstrumente informieren. sein muss. Voraussetzung ist eine Anstellung an einer Universitätsklinik über die gesamte Laufzeit des Programms.

Um sich für ein Clinician Scientist-Stipendium zu qualifizieren, muss der Bewerber seine wissenschaftliche Fokussierung mit Bezug auf die Innere Medizin anhand eines entsprechend außergewöhnlichen Forschungsprogramms und einschlägiger Vorarbeiten darlegen. Die Auswahl der Bewerber erfolgt nach objektiven Kriterien zur wissenschaftlichen Exzellenz der bereits erbrachten Forschungsarbeit (Publikationen, Drittmitteleinwerbungen) und zum wissenschaftlichen Profil des Bewerbers im Hinblick auf Motivation, Innovations- und Weiterentwicklungspotenzial.
Welche Vorteile ergeben sich aus der Teilnahme?

Durch die Clinician Scientist-Stipendien wird die grundlagenorientierte Forschung besser in den Ausbildungsweg zum Internisten integriert und es wird eine Garantie freier Forschungszeit zur Bearbeitung herausragender Forschungsprojekte geboten. Diese Zeit können die jungen Internisten nutzen, um weitere Forschungsgelder zu beantragen und ein eigenständiges wissenschaftliches Profil zu etablieren, ohne dabei ihre Facharztausbildung wesentlich zu verzögern. Die Fördermaßnahme soll somit die Facharztausbildung zum Internisten attraktiver gestalten und ideale Voraussetzungen 
für wissenschaftliches Arbeiten schaffen. Gleichzeitig sollen die Clinician Scientist-Stipendien als Grundlage für weiterführende Forschungsvorhaben dienen, um langfristig den Weg zur Habilitation und einer akademischen Laufbahn zu unterstützen.

Welche Problemfelder/Herausforderungen haben sich im Laufe der Jahre herauskristallisiert?

Es ist im Moment noch zu früh, diese Frage nachhaltig zu beantworten. Wir werden nun zunächst die nächste Förderungsperiode im Jahr 2018 abwarten und dann auch eine erste Evaluation der bereits geförderten Stipendiaten machen. Außerhalb des CSPs sind wir schon ein wenig weiter, weil die DGIM schon seit Längerem medizinische Doktoranden fördert, und können sagen, dass dieses Programm bereits zu vielen sehr guten Doktorarbeiten in der Inneren Medizin geführt hat.

\section{Professor Malek, vielen Dank für das Interview!}

Kontaktadresse: Prof. Dr. Nisar Malek, Medizinische Universitätsklinik Innere Medizin I, Otfried-Müller-Straße 10, 72076 Tübingen, Deutschland, Nisar.Malek@med.uni-tuebingen.de.

\section{Angaben zum Clinician Scientist-Programm (CSP) der Deutschen Gesellschaft für Innere Medizin e.V. (DGIM)}

Die DGIM schafft durch die Vergabe der drei Clinician Scientist-Stipendien ideale Voraussetzungen für wissenschaftliches Arbeiten und fördert dabei herausragende Forschungsprojekte - mehr Freiraum aufgrund von finanzieller Freiheit und Grundlage für weiterführende Forschungsvorhaben.

Voraussetzung für die Aufnahme in das Programm, das 2016 von der «Kommission Wissenschaft - Nachwuchsförderung» initiiert wurde, ist eine nachgewiesene wissenschaftliche Fokussierung mit Bezug auf die Innere Medizin bzw. ihre Schwerpunkte, welche durch ein entsprechendes außergewöhnliches Forschungsprogramm und einschlägige Vorarbeiten dokumentiert ist. Eine Teilnahme in der AG «Junge Internisten der DGIM» wird empfohlen. Das im Rahmen des CSP beantragbare Forschungsprojekt kann im Bereich der grundlagenorientierten, krankheitsorientierten oder patientenorientierten klinischen Forschung angesiedelt sein.

Die vollständigen Ausschreibungsunterlagen finden Sie hier: www.dgim.de $\rightarrow$ Nachwuchsförderung $\rightarrow$ Clinican Scientist-Programm.

\section{Ärztliche Schweigepflicht \\ Wann Ärzte Auskunft erteilen dürfen - und wann nicht}

Liegt eine gültige Schweigepflichtentbindung des Patienten vor, können Ärzte einem Betreuer oder der Polizei Auskunft erteilen. Ohne diese Entbindung ist die Rechtmäßigkeit sorgfältig im Einzelfall zu prüfen.

Im ärztlichen Alltag fallen viele hochsensible Daten an. Ein diskreter Umgang mit diesen Daten ist daher notwendig, um das Vertrauensverhältnis zwischen Arzt und Patient zu gewährleisten. Dies hat einen derart hohen Stellenwert, dass es durch § 203 des Strafgesetzbuchs und das medizinische Standesrecht geschützt wird. Bereits das Gelöbnis, das zu Beginn der Muster-Berufsordnung für die in Deutschland tätigen Ärztinnen und Ärzte abgedruckt ist, greift die ärztliche Schweigepflicht auf. Jeder Arzt hat die ihm «anvertrauten Geheimnisse auch über den Tod der Patientin oder des Patienten hinaus» zu wahren. Die Verpflichtung zur Verschwiegenheit ist jedoch nicht immer so einfach einzuhalten. Es gibt durchaus rechtlich legitimierte Ausnahmen, die ein Brechen der ärztlichen Schweigepflicht erfordern.

\section{Anfrage des Vormundes oder Betreuers}

Immer wieder erreichen Ärzte Anfragen mit der Bitte um Auskunft oder gar Akteneinsicht. Verlangt der Patient selbst Einsicht in seine Krankenakte, muss ihm dies in der Regel ohne Einschränkung gewährt werden, wenngleich er idealerweise in einem Arzt-PatientenGespräch Einsicht nehmen sollte. Auch wenn ein gesetzlich bestellter Vormund oder Betreuer mit diesem Anliegen an den Arzt heran- tritt, hat er ein Recht auf Akteneinsicht. Ist beispielsweise ein Volljähriger aufgrund einer psychischen Krankheit oder einer körperlichen, geistigen oder seelischen Behinderung nicht in der Lage, seine Angelegenheiten ganz oder teilweise zu erledigen, kann das Betreuungsgericht einen Betreuer als gesetzlichen Vertreter bestellen. Die Betreuung ist immer auf jene Aufgaben beschränkt, in denen sie erforderlich ist. Mögliche Aufgaben sind Vermögensfürsorge, Postkontrolle, Aufenthaltsbestimmungsrecht oder Gesundheitsfürsorge. Ein Betreuer hat nur Recht auf Auskunft oder Akteneinsicht beim Arzt, wenn die Auskunft für die Wahrnehmung

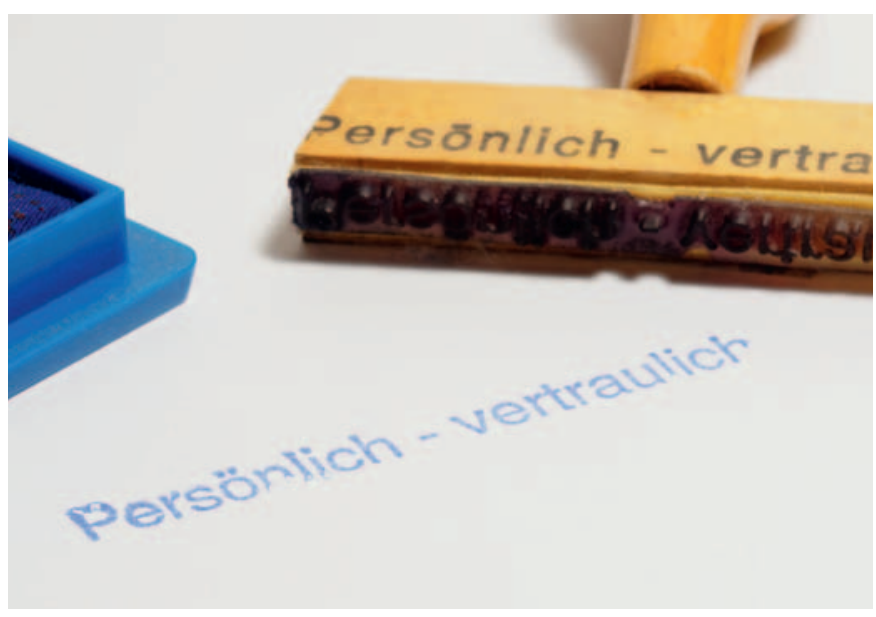


seiner konkreten Aufgabe nötig ist und zwar nur im erforderlichen Umfang. So benötigt ein Betreuer im Aufgabenkreis Vermögensfürsorge beispielsweise keine vollumfängliche Einsicht in die Patientenakte. Das Recht auf Auskunft eines Betreuers kann zusätzlich durch den Willen des Patienten eingeschränkt werden, sofern er einsichtsfähig ist. Eine Offenbarung von Patientengeheimnissen gegen den Willen eines einsichtsfähigen betreuten Patienten ist nicht zulässig. Daher sollte jede Anfrage eines Betreuers vorab sorgfältig geprüft werden.

\section{Anfrage von Ermittlungsbehörden und Gerichten}

Anfragen von Ermittlungsbehörden oder Gerichten erzeugen meist eine besonders hohe Unsicherheit. Man möchte einerseits keine personenbezogenen Daten unbefugt offenbaren, gleichzeitig jedoch die Ermittlungsarbeit der Behörden, wie die Suche nach vermissten Personen oder Unfallopfern, nicht behindern. Grundsätzlich folgt aus der strafrechtlich fixierten ärztlichen Schweigepflicht (\$ 203 StGB) ein Zeugnisverweigerungsrecht für Ärzte sowie deren Berufshelfer (§53 Abs. 1 Nr. 3 und $\S 53$ a StPO) gegenüber den Ermittlungsbehörden und vor Gericht.

Das im November 2015 in Kraft getretene Bundesmeldegesetz berechtigt Krankenhäuser jedoch dazu, gegenüber den zuständigen Behörden Auskunft in bestimmtem Umfang zu erteilen. Dieser umfasst Vor- und Familiennamen, Geburtsdatum und -ort (bei Geburten im Ausland auch den Staat), Staatsangehörigkeit, Anschrift sowie das Datum der Aufnahme und der Entlassung. Die Krankenhäuser dürfen Auskünfte jedoch nur erteilen, wenn dies zur Abwehr einer erheblichen und gegenwärtigen Gefahr, zur Verfolgung von Straftaten oder zur Aufklärung des Schicksals von Vermissten und Unfallopfern erforderlich ist. Den konkreten Grund für das Auskunftsersuchen muss die anfragende Behörde im Einzelfall darlegen. Eine vom Patienten verfügte Auskunftssperre greift in diesem Fall nicht. Jedoch dürfen Ärzte darüber hinaus auch gegenüber der Polizei, Staatsanwaltschaft oder den Gerichten keinerlei Auskünfte über Patienten erteilen. Anders verhält es sich, wenn der Patient den Arzt von der Verpflichtung zur Verschwiegenheit gegenüber der Polizei oder der Staatsanwaltschaft entbunden hat. Dann müssen Ärzte Auskunft erteilen.

Sie ist jedoch auf den in der Entbindungserklärung festgelegten Umfang zu beschränken. Schweigepflichtentbindungen von Angehörigen sind grundsätzlich unwirksam, selbst wenn der Patient bereits verstorben ist. Dennoch können Angehörige und Erben nach $\S 630 \mathrm{~g}$ Abs. 3 BGB ein Recht auf Einsicht in die Patientenakten haben.

\section{Wenn Gefahr im Verzug ist}

Das Zeugnisverweigerungsrecht wird durch das Beschlagnahmeverbot (§ 97 StPO) auf alle Aufzeichnungen, Mitteilungen und Gegenstände der ärztlichen Untersuchungsbefunde erweitert, die sich im Gewahrsam des Krankenhauses befinden. Dazu zählt auch die Patientenakte. Als Folge darf das Krankenhaus Unterlagen nur herausgeben, wenn entweder der Patient eine Entbindung von der Schweigepflicht vorgenommen hat oder die Ermittlungsbehörden einen Beschlagnahmebeschluss vorweisen, den in der Regel nur ein Richter ausstellen kann. Ausnahme: Wenn durch den Zeitverlust beim Einholen einer richterlichen Genehmigung Beweismittel verlorengehen können, ist «Gefahr im Verzug». Dann sind auch Polizei oder Staatsanwaltschaft berechtigt, eine Beschlagnahme anzuordnen.

Liegt ein gültiger Beschlagnahmebeschluss vor, müssen die Unterlagen im Original herausgegeben werden. Dabei gilt, unbedingt den genauen Umfang der Anordnung zu beachten, also auf welche Unterlagen sich diese konkret bezieht. Um die Patientensicherheit zu gewährleisten, sollten von allen Dokumenten Kopien angefertigt werden, bevor die Unterlagen herausgegeben werden. Zusätzlich sollten im Beschlagnahmeverzeichnis alle herausgegebenen Dokumente vollständig aufgeführt sein.

\section{Widerspruch gegen die Beschlagnahme}

Wenn die Ermittlungsbehörden keinen Beschlagnahmebeschluss vorlegen können, sollten Krankenhausmitarbeiter der Herausgabe ausdrücklich widersprechen und auf eine Dokumentation des Widerspruchs im Beschlagnahmeprotokoll bestehen. Fordern die Behörden dennoch die Herausgabe der Unterlagen, muss das Krankenhaus die Beschlagnahme dulden. Die Mitarbeiter sollten den Beamten dann lediglich zeigen, wo die Unterlagen lagern, diese jedoch nicht aktiv herausgeben.

\section{Originalquelle}

Kropp H, Günther U: Ärztliche Schweigepflicht: Wann Ärzte Auskunft erteilen dürfen - und wann nicht. Dtsch Arztebl 2017:114;48. (Direktlink zur Originalversion: www.aerzteblatt.de/archiv/195023/Aerztliche-Schweigepflicht-Wann-Aerzte-Auskunfterteilen-duerfen-und-wann-nicht)

Kontaktadresse: Dr. sc. hum. Uwe Günther, Sanovis GmbH, Richard-StraussStraße 69, 81679 München, Deutschland, info@sanovis.com.

\section{Medizinstudium \\ Urteil des Bundesverfassungsgerichts: Vergabe von Studienplätzen im Fach Humanmedizin ist teilweise verfassungswidrig - Statement bvmd}

Steigender Numerus Clausus und Wartezeiten, die länger als das Studium sind, stellen seit längerer Zeit Kritikpunkte am Zulassungsverfahren zum Medizinstudium dar. Nach der Verhandlung im Oktober verkündete das Bundesverfassungsgericht am 19. Dezember 2017 das mit Spannung erwartete Urteil bezüglich des Numerus Clausus in der Medizin. Nach Ansicht des Gerichts bedarf es Anpas- sungen verschiedener Aspekte des Auswahlverfahrens. Ungünstige Ortswahl darf nicht die Erfolgschancen auf einen Studienplatz reduzieren. Zudem müssen Landesunterschiede in den Abiturnoten einkalkuliert werden. Die Wartezeitquote ist praktisch nicht mehr zu halten und auch die Vergabe allein nach der Abiturnote ist nur in geringem Umfang verfassungskonform. 
Doch wie soll die Studierendenauswahl in der Medizin in Zukunft aussehen?

Bereits im vergangenen Juli legten der Medizinische Fakultätentag und die Bundesvertretung der Medizinstudierenden in Deutschland (bvmd) unter Berücksichtigung des Masterplans «Medizinstudium 2020» einen gemeinsamen Vorschlag für ein transparentes Modell der Studierendenauswahl der Medizin vor. Dieser wurde nach dem Urteil an die Vorgaben des Gerichtes angepasst [1].

Im neuen Zweistufenmodell sollen neben der Abiturnote fachspezifische Studierfähigkeitstests, Situational-Judgement-Tests sowie berufspraktische Erfahrung im medizinnahen Bereich als Auswahlkriterien einbezogen werden. Die Summierung dieser vier Kriterien ermöglicht eine bundesweite Reihung. Entsprechend dieser würden 50\% der Studienplätze unter Berücksichtigung der Ortspräferenz vergeben werden. Dabei soll die Ortspräferenz wieder zu einer echten Ortpräferenz werden und nur als nachrangiges Kriterium berücksichtigt werden. Die zweite Hälfte der Plätze wird im Auswahlverfahren der Hochschulen vergeben. Dieses orientiert sich an dem ersten, bundeseinheitlichen Verfahrensschritt. Es kann aber durch individuelle Test- und Interviewverfahren erweitert werden. Eine Standardisierung, wissenschaftliche Evaluation und eine im Verlauf zielgerichtete Anpassung der eingesetzten Auswahlkriterien ist dabei notwendig.

Nach dem Urteil des Bundesverfassungsgerichtes ist nun die Politik in der Verantwortung zeitnah neue Wege für eine evidenzbasierte, faire und transparente Zulassung zum Medizinstudium zu gehen.

\section{Literatur}

1 Medizinischer Fakultätentag, Bundesvereinigung der Medizinstudierenden in Deutschland e.V.: Zulassung zum Medizinstudium - teilweise verfassungswidrig. medizinische-fakultaeten.de/studium/themen/aktuelle-themen/zulassung (Zugriff 08.03.2018).

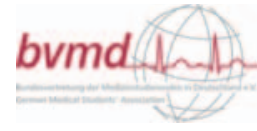

Kontaktadresse: Carolin Siech, Bundesvertretung der Medizinstudierenden in Deutschland e.V. (bvmd), Robert-Koch-Platz 7, 10115 Berlin, Deutschland, pr@bvmd.de. 